\title{
Cambios climáticos y aumento de la vegetación en la Península Fildes, Antártica.
}

Climate change and increase of vegetation in the Fildes Peninsula, Antarctica.

\section{Historial del artículo Recibido:}

07 de enero de 2019

Aceptado:

05 de junio de 2019.

\section{Carina Petscha,b; Maria Eliza Sotille ; Rafaela Mattos Costa ${ }^{\mathrm{b}}$; Katia Kellem da Rosa ${ }^{\text {b; Jefferson Cardia Simões }}{ }^{\text {b }}$}

a Departamento de Geociências, Universidade Federal de Santa Maria, Santa María, Brasil/ Centro Polar e Climático, Universidade Federal do Rio Grande do Sul, Porto Alegre, Brasil. Correo electrónico: carinapetsch@gmail.com.

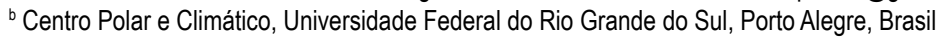

\section{Palavras chave}

Campos de musgos, NDVI, península Fildes

Keywords

Fildes Peninsula, Mosses fields, NDVI

\begin{abstract}
Resumo
O objetivo do artigo é avaliar a variação de área e localização dos campos de musgos na Península Fildes, Antártica, utilizando a técnica de NDVI e imagens LANDSAT, para o período 1986 - 2016, frente a cenários de mudanças climáticas. A técnica de NDVI foi aplicada em 7 datas de imagens LANDSAT e os produtos foram avaliados pelo índice Kappa. A área de vegetação aumentou de 1,5 para 2,7 km² no período. Os resultados do índice Kappa indicam a variação entre 0,81 a 0,93 de acurácia, com mais de $70 \%$ das áreas mapeadas indicadas acima de 0,1 (área muito provável de vegetação). Os maiores campos de musgos ( $83 \%$ daqueles de maior dimensão) se localizam na porção orientada para o estreito de Drake e estão associados à umidade e vento proveniente do oceano. Há maior quantidade de matéria orgânica e formação de alagados nos paleovales em "U" voltados para o Drake, contribuindo para a fixação da vegetação. Os campos de musgos continuarão a aumentar ou haverá modificação da sua localização diante de cenários de retração da geleira local Collins e o consequente aumento da disponibilidade de água líquida e sedimentos no ambiente proglacial e periglacial.
\end{abstract}

\section{Abstract}

The objective of the article is to evaluate the variation of area and location of mosses in the Fildes Peninsula, Antarctica, using the NDVI technique and LANDSAT images, for the period 1986-2016, in the face of climate change scenarios. The NDVI technique was applied on 7 LANDSAT images and the products were evaluated by the Kappa index. The vegetation area increased from 1.5 to $2.7 \mathrm{~km}^{2}$ in the period. The Kappa index results indicate variations with 0,81 to 0,93 accuracy, more than $70 \%$ of the mapped area were indicated above 0.1 (most likely vegetation area). The largest mosses fields ( $83 \%$ of the larger ones) are located in the part facing the Drake Passage and are associated to the moisture and wind coming from the ocean. The greatest amount of organic matter and floodwaters occur in the "U" valleys facing the Drake, contributing to the vegetation fixation. The mosses fields will continue to increase in the face of scenarios point to increasing mean air temperature and retraction of the local Collins glacier and, consequently, an increase in the availability of liquid water and sediments in the proglacial and periglacial environment. 


\section{Introdução}

De acordo com Amesbury et al. (2017) existe um padrão generalizado de resposta biológica ao aumento de temperatura na Península Antártica (PA). Com a retração de muitas geleiras da PA(Cook, Fox, Vaughan, \& Ferrigno, 2005, Cook et al., 2016) haverá uma transformação da paisagem, além de colonização por plantas macroscópicas, em áreas recentemente expostas (Smith, 2001; Amesbury et al., 2017). Ressalta-se que a PA está passando por uma das fases mais rápidas de aquecimento da Terra, nas últimas décadas (Mulvaney et al., 2012) na ordem de $3,0^{\circ} \mathrm{C}$ entre 1961 e 2000 (Steig et al., 2009).

Para a ilha Rei George o aquecimento regional resultou em rápidas mudanças ambientais, como a deglaciação, que representa uma grande ameaça para o ecossistema local (Rakusa-Suszczewski, 2003). Vários estudos evidenciam a retração de geleiras, especificamente na ilha Rei George relacionada ao aumento da temperatura do ar na região da PA (Bremer, 1998; Park, Chang, Yoon, \& Chung, 1998; Simões, Bremer, Aquino, \& Ferron, 1999; Braun \& Gossmann, 2002; Rosa, Vieira, Ferrando, \& Simões, 2009; Rückamp, Braun, Suckro, \& Blindow, 2011) para períodos entre 1950 e 2015. A retração de geleiras representa maior área para desenvolvimento de campos de musgos na área. A comunidade de musgos está bem desenvolvida para a região, mas é restrita a lagos derretidos, fluxos de derretimento e outros locais onde a água livre está disponível pelo menos em parte do verão (novembro - março) para realizar fotossíntese e crescimento (Schaefer et al., 2004). O alto número de espécies e também ocorrência individual para cada espécie são provavelmente associadas a condições climáticas úmidas e quentes da ilha Rei George (Putzke, Athanásio, de Albuquerque, Victoria, \& Pereira, 2015).

Entender os fatores que influenciam na distribuição da vegetação de áreas livres de gelo é uma ferramenta para compreender os efeitos das mudanças climáticas (Poelking, Andrade, Schaefer, Simas, \& Fernandes Filho, 2011) e o Sensoriamento Remoto tem contribuído significantemente para o mapeamento da vegetação (Chilar, 2000). Quanto à metodologia utilizada, o NDVI (Índice de Vegetação por Diferença Normalizada) quantifica a vegetação pela diferença da resposta espectral dessa à radiação solar em diferentes faixas do espectro eletromagnético, onde a absorção pela clorofila e a estrutura celular das folhas resulta em baixa reflectância nos comprimentos de onda do visível e alta reflectância no comprimento de onda do infravermelho (Billings \& Morris, 1951; Knipling, 1970). Essa técnica já foi utilizada com êxito em várias áreas da Antártica (Fretwell, Convey, Fleming, Peat, \& Hughes,
2011; Poelking et al., 2011; Andrade et al., 2015; Sotille, Bremer, \& Simões, 2017; Andrade et al., 2018). Para a área da ilha Rei George Poelking et al. (2011) realizaram a classificação da vegetação para a península Potter utilizando o NDVI associado à classificação supervisionada; para a península Fildes Medeiros et al. $(2014 ; 2018)$ associaram a vegetação, mapeada com a técnica de NDVI, com a incidência da radiação solar; Sotille et al. (2017) utilizaram a técnica de NDVI para a Baía Esperança; e Fretwell et al. (2011) utilizaram o NDVI para identificar áreas de vegetação na Península Antártica.

O objetivo do artigo é avaliar a variação de área e estabelecer padrões de localização dos campos de musgos na Península Fildes, Antártica, utilizando a técnica de NDVI e imagens LANDSAT, para o período de 1986 -2016, frente a cenários de mudanças climáticas.

\section{Caracterização da área}

A ilha Rei George (figura 1) é a maior das Shetlands do Sul, localizada no extremo norte da Península Antártica, com área total de aproximadamente $1250 \mathrm{~km}^{2}$, dos quais mais de $90 \%$ estão cobertos por neve (Osmanoğlu, Braun, Hock, \& Navarro, 2013) e está dividida em vários domos de gelo conectados (Simões et al., 1999). Na maioria das áreas livres de gelo dessa ilha, a água de derretimento das geleiras é descarregada em pequenos canais, dos quais muitos fluem até lagos. Estes lagos se formam principalmente perto das margens das geleiras, ou em áreas planas de plataformas marinhas de abrasão soerguidas (Hochschild \& Staeblein, 1998; Mäusbacher, 1991). A vegetação na ilha é composta basicamente por tundras mal desenvolvidas e consiste quase exclusivamente em criptógamas, líquens e musgos, com duas espécies de Deschampsia antartica e Colobanthus quitensis (Olech \& Assalski, 2001).

A península Fildes (figura 1), localiza-se na parte sul da ilha Rei George $\left(62^{\circ} 08^{\prime}\right.$ e $6^{\circ} 14^{\prime}$ S; $59^{\circ} 02^{\prime}$ e $58^{\circ} 51^{\prime}$ O) e é limitada a nordeste por um pequeno domo de gelo, a geleira Collins (figura 1). Essa geleira localiza-se aproximadamente na latitude $62^{\circ} 12^{\prime} \mathrm{S}$ e longitude $58^{\circ} 57^{\prime} \mathrm{W}$, com uma área de $15 \mathrm{~km}^{2}$ e elevação máxima de $270 \mathrm{~m}$ e término atual em terra (Simões, da Rosa, Czapela, Vieira, \& Simões, 2015). Rosa et al. (2015) indicam que o processo de retração da geleira produz distintos ambientes na parte norte da península Fildes, com fluxos de fusão provenientes da geleira, originando lagos e alagados com dois tipos de drenagens: um interno e outro voltado para a enseada Maxwell (Rosa et al., 2015). 


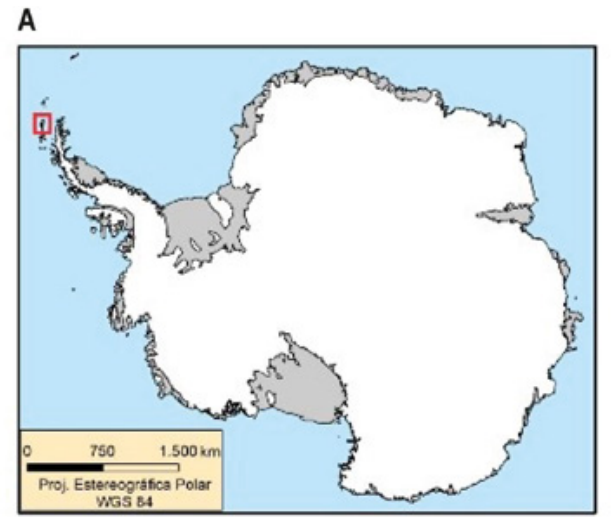

B

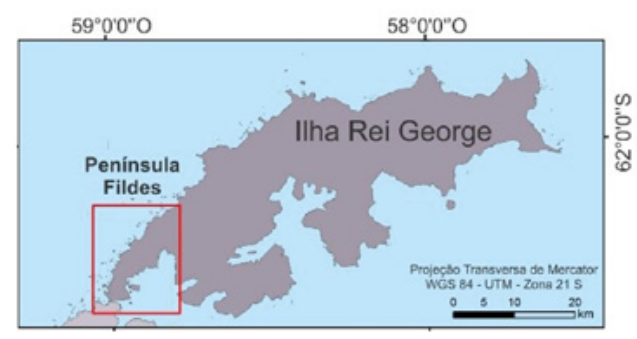

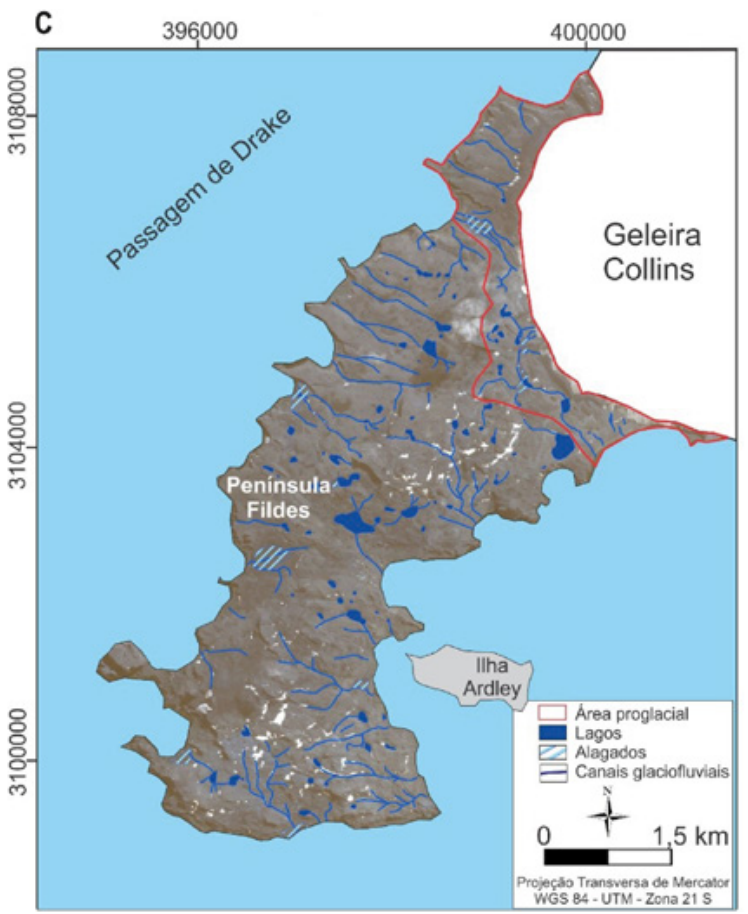

Figura 1. (A) Localização da Antártica com polígono vermelho destacando a ilha Rei George; (B) Ilha Rei George com polígono vermelho em destaque para a península Fildes; (C) Península Fildes e geleira Collins. Fuente: Elaboración propia (2019).

De uma forma geral, Bremer (2008) afirma que as áreas livres de gelo das ilhas Shetland do Sul são compostas por terrenos jovens e drenados por canais intermitentes e a morfodinâmica está associada a processos físicos e químicos devido ao derretimento da neve e do gelo. De acordo com Michel et al. (2014), os solos da península Fildes são bem desenvolvidos para padrões antárticos, sendo os criossolos dominantes relacionados a crioturbação e processos da camada ativa. Os processos periglaciais são dominantes em elevação acima de $50 \mathrm{~m}$ e onde são comuns solos em padrão. Os processos geomorfológicos que ocorrem atualmente na península Fildes são nivação, solifluxão, ablação e erosão e crioturbação (Simas et al., 2008). López-Martínez, Serrano, Schmid, Mink, \& Linés (2012) identificaram as seguintes formas na península Fildes: plataformas marinhas soerguidas, vales planos, solos com padrão e lóbulos de gelifluxão.

\section{Materiais e métodos}

Foram utilizadas 7 imagens Landsat, sensores TM 4 e 5, ETM+ 7 e OLI/TIRS 8 do período 1986-2017. Devido à alta cobertura de nuvens, característica da região, não foi possível obter imagens com maior frequência, contudo se optou por utilizar somente imagens desses sensores para que escala de trabalho fosse a mesma. As datas utilizadas foram: 01/03/1986; 27/02/1988; 28/01/1989; $19 / 01 / 2003 ; 17 / 03 / 2015 ; 29 / 04 / 2016$. As imagens foram georreferenciadas com base no mosaico de imagens Landsat da Antártica (LIMA) no software QGIS 2.18.9. Foi feita a correção atmosférica nas bandas necessárias para o cálculo e os valores foram convertidos de DN (Número digital) para reflectância no topo da atmosfera.

A técnica de NDVI foi escolhida devido os resultados satisfatórios obtidos em várias áreas livres de gelo da Antártica (Fretwell et al., 2011; Poelking et al., 2011; Andrade et al., 2015; Sotille et al., 2017; Andrade et al., 2018). O NDVI é baseado na razão entre os comprimentos de onda do vermelho (Red) e infravermelho próximo (NIR) através da fórmula: $\mathrm{NDVI}=(\mathrm{NIR}-\mathrm{Red}) /(\mathrm{NIR}+\mathrm{Red})$. Para o Landsat 8 os intervalos do infravermelho próximo e do vermelho representam as bandas 5 e 4 , respectivamente, já para o Landsat 4, 5 e 7 representam as bandas 4 e 3 .

No índice, é atribuído para cada pixel da imagem um valor que varia de $-1 \mathrm{a}+1$. Valores negativos de NDVI, próximos de -1 , correspondem a áreas com presença de neve ou de água, valores próximos de zero normalmente correspondem $\mathrm{a}$ áreas de solo exposto e valores próximos a +1 indicam pixels com conteúdo muito alto de clorofila (Fretwell et al., 2011). Assim, para definir as áreas consideradas com 


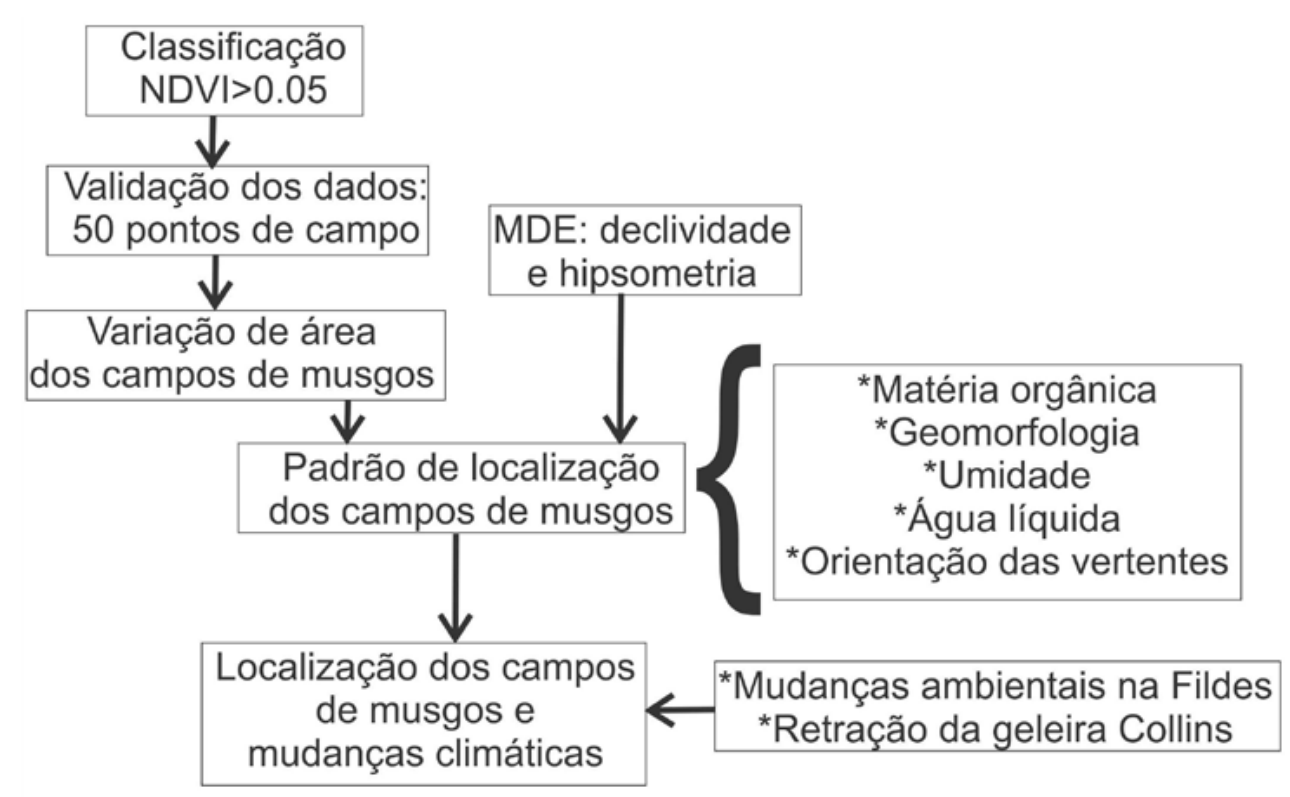

Figura 2. Fluxograma representando a metodologia utilizada, validação dos dados, padrão de localização dos campos de musgos e possível comportamento futuro diante de mudanças climáticas e ambientais.. Fuente: Elaboración propia (2019).

presença de cobertura vegetal foi estabelecido um limiar maior que 0,05 , já que de acordo com Fretwell et al. (2011) e Casanovas, Black, Fretwell, \& Convey (2015) áreas que apresentam NDVI inferior a isso são improváveis de serem vegetadas para a Antártica. Além disso, foi aplicado um filtro para eliminar pixels isolados que foram classificados como de possível presença de vegetação.

Com a aplicação do limiar e a reclassificação do NDVI foi possível obter as áreas com campos de musgos e foi estabelecido um padrão de localização quanto a presença de matéria orgânica, geomorfologia, umidade, água líquida e orientação das vertentes, (figura 2) segundo o método de Vera et al. (2013), Victoria et al. (2013) e Ruiz-Fernández, Oliva, \& García-Hernández (2017). O índice Kappa foi utilizado para avaliar a acurácia dos resultados, baseado em 50 pontos obtidos em campo com uso de GPS (Global Positioning System). Os trabalhos de campo foram realizados nos verões de 2015 e 2016.

O Modelo Digital de Elevação (MDE) utilizado foi produzido por Braun, Betsch, \& Seehaus (2016), com resolução espacial de $12 \mathrm{~m}$, para toda a ilha Rei George a partir de imagens TanDEM-X, de janeiro de 2012, referenciadas e validadas com base em medidas DGPS. Os dados foram processados utilizando interferometria e um MDE antigo como referência (Braun et al., 2016).
Para a interpretação geomorfológica foram elaborados mapas de hipsometria e declividade no software QGIS 2.18. A declividade foi delimitada com as classes de porcentagem entre: $0 \%-3 \%$ (plano), $3 \%-8 \%$ (suave ondulado), $8 \%-20 \%$ (ondulado), $20 \%-45 \%$ (forte ondulado), $45 \%$ - 75\% (montanhoso). A hipsometria foi aplicada entre 0 - $300 \mathrm{~m}$ com intervalos definidos de $50 \mathrm{~m}$.

A partir do cenário de retração da geleira Collins e mudanças ambientais já observadas na península Fildes será analisada o possível comportamento futuro dos campos de musgos para a área de estudo com base no padrão de localização atual (figura 2).

\section{Resultados}

A área dos campos de musgos foi calculada nas imagens que não apresentavam nenhuma porção coberta por nuvem e excluída a imagem de 2017 por apresentar cobertura de neve em toda porção central e sul da península Fildes. Aárea aumentou de 1,50 para 2,73 $\mathrm{km}^{2}$ no período 1986 - 2016 (figura 3). A taxa de elevação de área para todo o período foi de 0,041 . As áreas que mais apresentam crescimento da vegetação estão associadas à locais com provável aumento de água líquida, principalmente em paleovales em U (fotografias 1,4 e 5 ; figura 4 ) e próxima à geleira Collins. O aumento da vegetação próximo a zona proglacial 

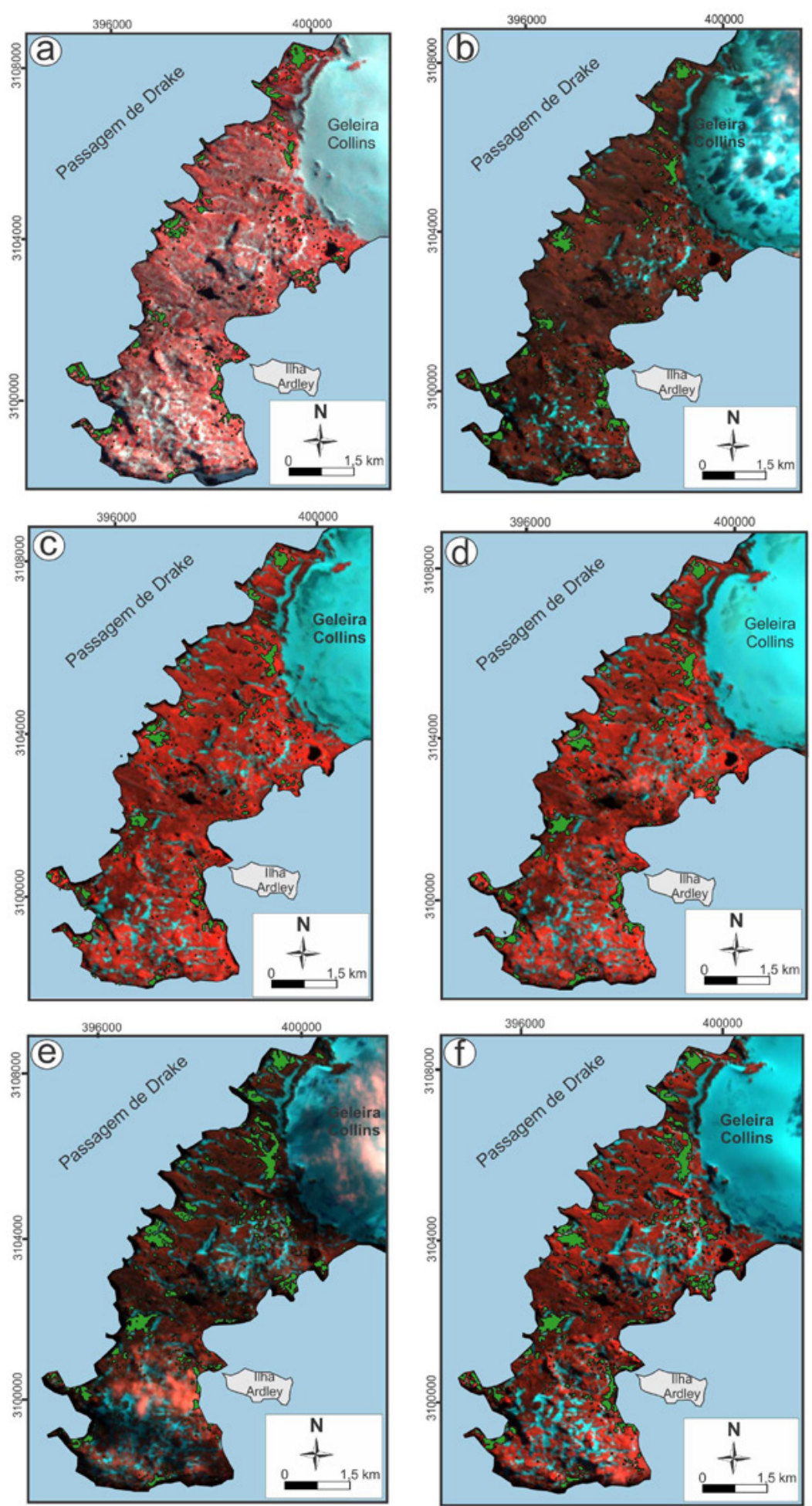

\section{Legenda}

Classificação NDVI $>0.05$

Figura 3. Resultados do NDVI para as imagens Landsat da península Fildes. Os maiores campos de musgos, descritos no item 4.2, já estavam presentes na imagem de 1986 e a partir disso aumentaram em área e também outras áreas foram colonizadas. A representa a imagem de 01/03/1986; B-27/02/1988; C-28/01/1989; D-19/01/2003; E-17/03/2015; F-29/04/2016. Fuente: Elaboración propia (2019). 

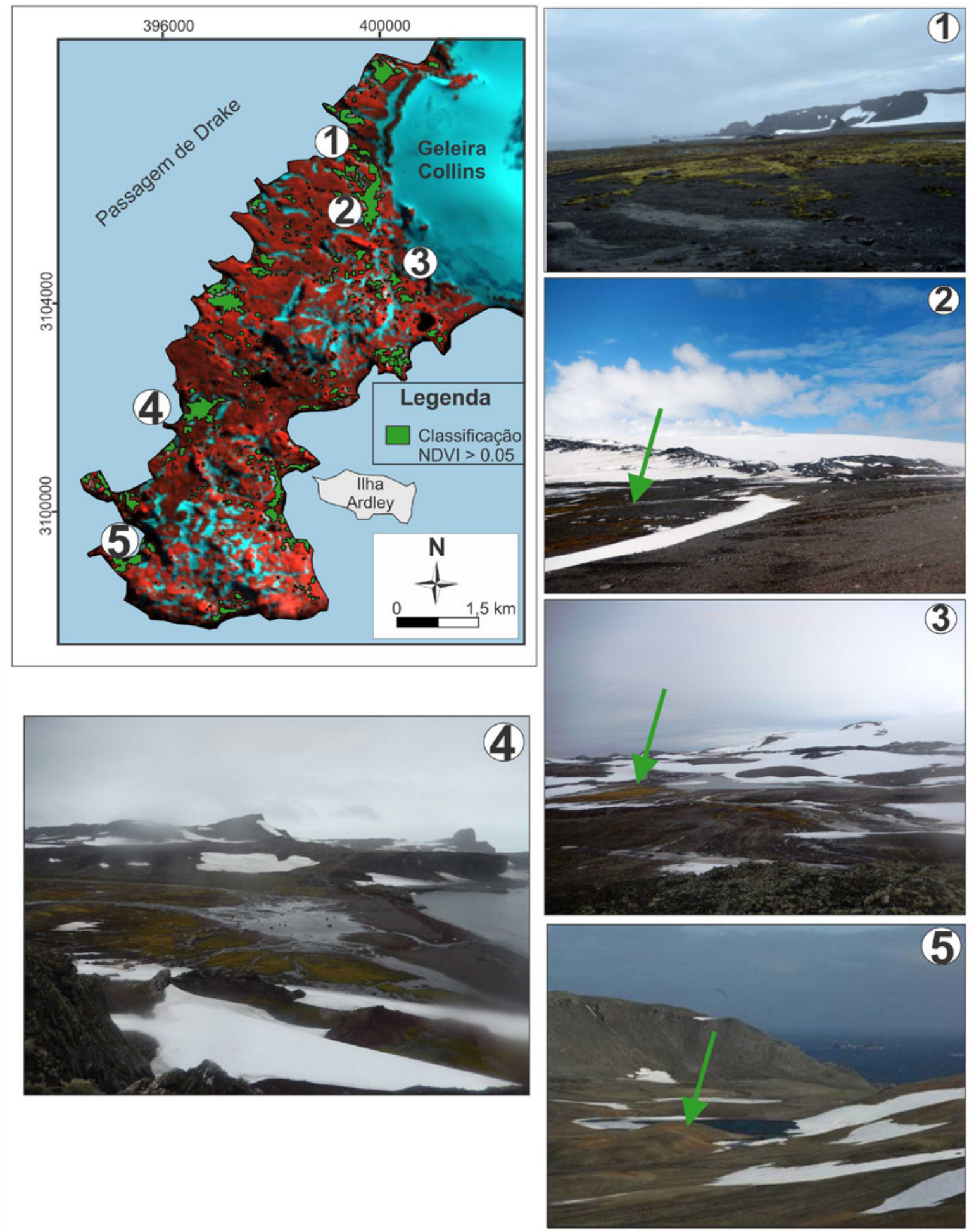

Figura 4. Fotografia 1; campo de musgos em paleovale em U. Fotografia 2; campo de musgos amarelado, na zona proglacial. Fotografia 3; campo de musgos com mais clorofila, na zona proglacial. Fotografia 4; campo de musgos em paleovale em U. Fotografia 5; campo de musgos amarelado em paleovale em U. A imagem classificada é de 26/04/2016 e as setas em verde indicam os campos de musgos. Fuente: Elaboración propia (2019). 


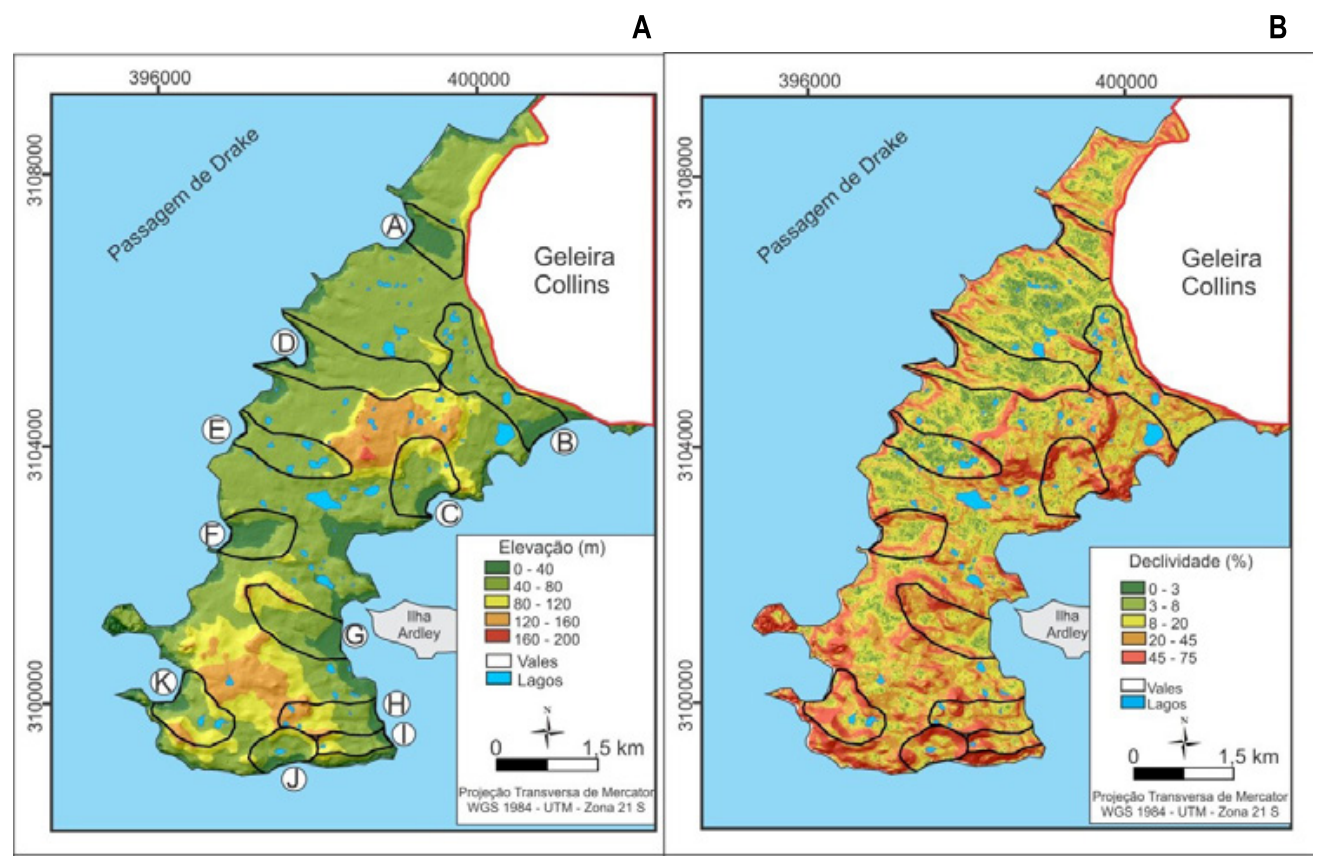

Figura 5. Relevo da península Fildes: A) Hipsometria; B) Declividade. A sequência de letras no mapa A (elevação) representa a nomenclatura atribuída aos vales. Observa-se que as áreas que se localizam os maiores campos de musgos são paleovales em U. Fuente: Elaboración propia (2019).

pode estar associado à retração da geleira já indicada por Simões et al. (2015) e o aumento da quantidade de água líquida no sistema (será detalhado adiante).

O índice Kappa para verificação da acurácia da classificação NDVI variou entre 0.81 e 0.93 , sendo a menor para a imagem de 2015 e o maior em 1989 e 2003. Apesar de terem sido consideradas como áreas prováveis de vegetação zonas acima de 0,05 , ressalta-se que considerando as 7 imagens, mais de $70 \%$ das áreas indicadas estão acima de 0,1 (área muito provável de vegetação) e $25 \%$ das áreas estão acima de 0,2 (área praticamente certa ser de vegetação). Andrade et al. (2018) chegaram a um valor de índice Kappa de 0,73 utilizando o NDVI para mapeamento de vegetação na Península Fildes.

Considerando os maiores campos de musgos (figura 4) a técnica de NDVI delimitou com exatidão próxima de $90 \%$ as áreas de campos de musgos com lâmina de água e condições mais saudáveis das plantas (fotografias 1, 3 e 4). Os musgos que formam os campos que ocupam a área representada pelas fotografias 2 e 5 se localizam em áreas com lâmina de água menos espessa e relevo plano sem formação de wetlands, e possuem aspecto mais amarelado.

\section{Discussões}

Técnica de NDVI e uso de imagens Landsat:vantagens e desvantagens

A maior parte da vegetação mapeada neste estudo são de campos de musgos, havendo uma possibilidade de haver líquens ou algas. Andrade et al. (2018) utilizaram o NDVI, GNDVI (Green NDVI) e classificação com amostras de campo e conseguiram mapear além de campos de musgos áreas com líquens, contudo utilizaram imagens Quickbird. Fretwell et al. (2011) ressaltam que os líquens, onde a morfologia estrutural elimina a clorofila contida em suas células, a possibilidade de identificação através de Sensoriamento Remoto é reduzida. Casanovas et al. (2015) ressaltam que a técnica NDVI para a deteç̧ão de vegetação pode ignorar a presença de liquens, mesmo que a cobertura da terra seja extensa. De acordo com as verificações feitas em campo e comparação com o mapeamento, os campos de musgos são a maioria das áreas mapeadas devido também a sua escala espacial. As áreas de líquens, na sua maioria, são menores que 30 metros, ou seja, justamente o tamanho do pixel das imagens Landsat utilizadas.

Os resultados do mapeamento dos campos de musgos, considerando a escala espacial adotada, são satisfatórios 
e próximos aos encontrados por Fretwell et al. (2011) que utilizaram 13 imagens Landsat ETM para a Terra de Graham, na parte norte da península Antártica e Johansen \& Karlsen (2005) que utilizaram imagens Landsat para o Ártico O ideal seria o uso de outras imagens de melhor resolução espacial contudo, devido a presença de nuvens não é possível obter uma escala temporal que permita a associação com mudanças ambientais na península Fildes. As imagens MODIS apresentam resolução espacial (500m) que impediria o mapeamento de qualquer um dos campos de musgos classificados com as imagens Landsat devido ao tamanho do pixel imageado. As imagens Sentinel 2 (resolução espacial de $10 \mathrm{~m}$ ) apresentam grande potencial para o mapeamento de vegetação na área da Antártica, porém somente uma imagem sem nuvens está disponível para a área da península Fildes e infelizmente é de dezembro, período em que há cobertura de neve na área impossibilitando a classificação da vegetação.

Ainda que os campos de musgos possuam certa heterogeneidade sendo um alvo com presença de lâminas de água e alguns locais com vegetação mais amarelada e em outros mais esverdeada, o índice Kappa mostrou que a técnica NDVI delimitou com exatidão mais de $70 \%$ dos campos de musgos como muito prováveis de serem vegetação. Comparando com os resultados de NDVI obtidos em Fretwell et al. (2011) e Andrade et al. (2018) o resultado está adequado.

Outro fator que pode influenciar nos resultados obtidos é a sazonalidade a que essa vegetação está submetida ficando sem cobertura de nuvens somente nos meses de verão. Ainda assim, a cobertura de neve na península Fildes é variável de acordo com a temperatura média do ar, dias com temperatura acima de $0^{\circ} \mathrm{C}$ e precipitação de chuva líquida ou por neve. Assim, o recomendado é que as imagens de satélite sejam do final do verão, por volta do final de fevereiro e março. Contudo, infelizmente a disponibilidade de imagens para a área e utilizada nessa pesquisa variou do final de janeiro até o final de abril o que pode influenciar nos resultados obtidos.

\section{Localização dos principais campos de musgos}

Alguns dos fatores ambientais que afetam o crescimento da vegetação na Antártica são, de acordo com Jawak et al. (2016), água disponível, riqueza de nutrientes, exposição a ventos, spray marinho e solos. Para o caso da península Fildes, dos 6 maiores campos de vegetação encontrados com a técnica de mapeamento por NDVI, 5 encontram-se na porção voltada para o estreito de Drake. Os fatores a que isso se deve são, provavelmente:
Matéria orgânica. Há colônias de pinguins, skuas e petrels abandonadas e ativas na região costeira, além de outros animais que promovem transferência de nutrientes para essas áreas contribuindo para crescimento dessa vegetação (Michel et al., 2014). Há pelo menos duas áreas de praias, na porção voltada para o estreito de Drake em que a presença de animais como focas, lobos e elefantes marinhos foi observada e coincidem com as áreas de maior concentração de musgos;

Localização geomorfológica. As grandes áreas de vegetação se localizam em zonas classificadas como terraços marinhos ou paleovales em forma de "U" (Petsch, 2018), principalmente nas porções mais planas dos vales é comum encontrar alagados que proporcionam a fixação da vegetação. Para a Ponta Elefante, Ruiz-Fernandez et al. (2017) também encontraram o padrão de campos de musgos em áreas alagadas. De acordo com Victoria et al. (2013), os campos de musgos ocorrem nessas áreas pois possuem criossolos hidromórficos estáveis. Segundo a classificação geomorfológica de López-Martínez et al. (2012), um dos maiores campos de musgos está localizado justamente sobre áreas de solo em padrão (fotografia 2; figura 4). O campo de musgos localizado sobre a área de solo em padrão (Fotografia 2; figura 3 ) responde com reflectância diferenciada indicando a coloração amarelada. A presença de feições padronizadas no solo, como círculos de pedra e linhas de sedimentos que dependem do acúmulo de materiais finos em seu centro, favorecem a colonização da vegetação por líquens em locais secos e ventosos e por musgos em áreas alagadas (Serrano, 2003). É provável que esse comportamento deixe a área menos úmida, e modifique o comportamento dos musgos na área.

Umidade proveniente do estreito de Drake. Ventos mais frequentes são provenientes de Noroeste e Oeste e em fevereiro são mais comuns trazendo precipitação, umidade relativa e cobertura de nuvens (Rakusa-Suszczewski et al., 2003). Devido essa condição de vento, ocorre provavelmente entrada de umidade na Península, proveniente do estreito de Drake que acaba contribuindo para o crescimento da vegetação;

Água líquida disponível. Como um efeito do aumento da temperatura do ar para a região, ocorre mudança na disponibilidade de água, expressa pelo aumento nas precipitações, especialmente durante o verão antártico (Turner, Colwell, \& Harangozo, 1997), e derretimento do gelo das geleiras;

Orientação das vertentes. Os setores de acumulação de neve que perduram por mais tempo no verão estão inseridos 
junto às vertentes orientadas para o sul na Península. Dessa maneira, os campos de vegetação voltados para o Norte dispõem de mais tempo para crescimento e desenvolvimento no período de verão. Andrade et al. (2018) ressaltam que a quantidade de radiação incidente é um elemento determinístico no desenvolvimento da vegetação para a península Fildes.

Apesar de terem sido evidenciadas características ambientais que refletem o padrão de fixação e crescimento do campo de musgos, alguns fatores que poderiam ser fundamentais para a localização não apresentam influência direta. A vegetação na península da Ponta Elefante tem sua distribuição espacial condicionada claramente pelo processo de recuo da geleira Rotch, mostrando o tempo como um fator-chave (Ruiz-Fernández et al., 2017). Uma possível explicação para isso é o tempo que a Fildes é considerada como área livre de gelo. A partir de $10.000 \mathrm{AP}$, quando se iniciou o processo de desnudação da península, o campo de gelo da ilha Rei George retraiu, deixando o sistema glacial do domo Collins e pequeno campo de gelo nas áreas planálticas no Norte e Sul (John, 1972). Ou seja, os processos periglaciais e retrabalhamento do relevo pelos canais de fusão da neve se tornaram mais importantes que a distância em relação à geleira.

\section{Características da vegetação}

Em dois campos de musgos (figura 3; fotografias 2 e 5), foi observada coloração amarelada denotando folhas com menos clorofila. Essas mudanças na coloração dos musgos de verde para vermelho-marrom é provavelmente uma resposta ao estresse, que reflete a fase de transição entre musgo vivo e doente (Lucieer, Robinson, Turner, Harwin, \& Kelcey, 2012). O estresse da planta para essa área da península Fildes está, provavelmente, ligado à ausência de acumulação de água na área do campo de musgo, solo menos espesso, condições do relevo e maior insolação.

Para o caso do campo de musgos da fotografia 5, localizado numa área de fundo de vale com água líquida disponível, provavelmente o fator relevo contribuí para haver menos insolação na área ou então solo pouco espesso. Por outro lado, a área pode também ter alta quantidade de matéria orgânica,contribuindo para a manutenção da vegetação nesse vale. O campo de musgo da fotografia 2 (figura 3 ) está numa área onde há passagem de água vinda degelo da geleira, porém não há acumulo e formação de alagados nessa área. Esse campo de musgos está sobre uma área de solo em padrão (López-Martínez et al., 2012), isso pode dificultar o pleno desenvolvimento da planta. Para musgos da Antártica, as características de reflectância variam entre as espécies e são significativamente influenciadas pelas condições ambientais sob as quais as plantas crescem, como diferenças no conteúdo de água eo fato do musgo estar crescendo numa crista ou vale (Bubier, Rock, \& Crill, 1997).

O espectro de reflectância dos musgos é bastante similar ao das plantas vasculares, sendo que diferentes espécies podem apresentar reflectância distintas na região do infravermelho próximo - NIR (Haselwimmer \& Fretwell, 2009). Musgos saudáveis mostram alta refletância no infravermelho próximo, musgos desidratados apresentam contraste menor entre as regiões do vermelho e do NIR (Lucieer et al., 2012). Salienta-se que para os campos de musgos das fotografias 1, 3 e 4 (figura 4) a vegetação apresenta-se esverdeada caracterizando plantas saudáveis.

\section{Mudanças ambientais na península Fildes e localização da vegetação}

O aumento da vegetação na península Fildes segue o padrão da Península Antártica para áreas deglaciarizadas, quase dobrando no período 1986 - 2016. Também condiz com outros estudos na península Fildes como Gerighausen, Bräutigam, Mustafa, \& Peter (2003) e Peter, Bußer, Mustafa, \& Pfeiffer (2007), que relataram um aumento total da população de D. antarctica, com um aumento no número de plantas de 14 para 160 durante as últimas décadas.

Com o aumento da temperatura e da disponibilidade de água nesses sistemas é provável que os campos de musgos ocupem outras áreas da península Fildes no futuro próximo. Mesmo havendo uma tendência de resfriamento em algumas áreas da Península Antártica (segundo Turner et al., 2016, a tendência da temperatura atmosférica da PA nas últimas três décadas passou de aquecimento de $0,32^{\circ} \mathrm{C} /$ década em $1979-1997$ para resfriamento de $-0,47^{\circ} \mathrm{C} /$ década em 1999 - 2014), a ilha Rei George apresenta aumento de temperatura. Kejna, Araźny, \& Sobota, (2013) aponta que para a ilha Rei George houve um aumento de temperatura de $0,19^{\circ} \mathrm{C} / 10$ anos $(\mathrm{r}<0,05)$ durante o período 1948 - 2011. De acordo com Pudelko, Angiel, Potocki, Jedrejek, \& Kozak (2018), a taxa do aumento variou de ano para ano, com o maior aquecimento ocorrendo nos meses de inverno $\left(0,36^{\circ} \mathrm{C} / 10\right.$ anos). Também é possível observar que as mudanças climáticas afetaram diretamente o crescimento e localização da vegetação para a área de estudo, como já indicado por vários outros autores para a Antártica (Convey, 2003; Convey \& Smith, 2006; Pritchard \& Vaugan, 2007; Green, Sancho, Pintado, \& Schroeter, 2011). 
A maior parte dos paleovales em "U" (fotografias 1, 4 e 5; figura 3) estão passando por um processo de diminuição do grau de entalhamento. Esses vales estão sendo preenchidos por sedimentos provenientes das paredes das falésias e também transportados por canais de fusão da neve. A maioria dos vales se encaixa na classe de baixa dimensão interfluvial e estão evoluindo de um grau de entalhamento muito forte $(>160 \mathrm{~m})$ para médio $(40 \mathrm{~m}-80 \mathrm{~m})$. O preenchimento dos vales irá alterar a dinâmica de localização dos canais glaciofluviais modificando a alimentação dos wetlands que são importantes condicionantes para o crescimento dos campos de musgos.

As mudanças climáticas para a Antártica, especialmente o aumento das temperaturas, resulta em maior disponibilidade de água e levará a (i) aumento das taxas de colonização local e de longa distância, e (ii) expansão da população em escala local, levando a (iii) maior diversidade terrestre, biomassa e complexidade trófica, (iv) estrutura ecossistêmica mais complexa (Singh, Singh, \& Khare, 2018). Para o caso da geleira Collins, Simões et al. (2015) indicam sua retração nas últimas décadas e Rückamp et al. (2011) indicam o quase total desaparecimento da geleira para os próximos 200 anos. Diante desse cenário de continuidade na retração da geleira Collins é importante manter esse banco de dados de mapeamento temporal da vegetação da península Fildes atualizado e técnicas como o NDVI são fundamentais para esse propósito. Para monitorar e avaliar futuras mudanças na vegetação da Península Antártica, é necessária uma avaliação quantitativa robusta da vegetação para a região (Fretwell et al., 2011).

\section{Considerações finais}

De acordo com esse mapeamento a área com vegetação cresceu de 1,5 para 2,73 $\mathrm{km}^{2}$ no período 1986 - 2016, mas provavelmente o mapeamento só abrangeu áreas com musgos, pois os líquens não apresentam grande quantidade de clorofila em suas células limitando sua capacidade de deteç̧ão. A técnica de NDVI mostrou resultados adequados com índice Kappa variando entre $81 \%$ e $93 \%$ com mais de $70 \%$ onde as áreas indicadas estão acima de 0,1 (área muito provável de vegetação).

Foram encontradas duas áreas com campos de musgos amarelados, indicando estresse da planta que pode estar ligado à não formação de alagados (os quais mantém a umidade para essas briófitas). Os dois campos de musgos podem estar localizados, ainda, em áreas com solos pouco espessos, áreas rochosas e com muita insolação. Para um dos campos de musgos, provavelmente, a alta presença de matéria orgânica pode ser o responsável pela manutenção da vegetação nessa área.

É importante determinar quais fatores controlam o crescimento da vegetação na península Fildes, já que algumas variáveis ambientais estão rapidamente sendo modificadas por mudanças climáticas. Ocorre um aumento da disponibilidade de água líquida para a área, proveniente da retração da geleira Collins e também do aumento de precipitação líquida, resultado do aumento da temperatura atmosférica nas últimas décadas. O relevo contribui para o acúmulo de água em áreas planas dos vales em formato de "U" dando origem aos maiores campos de musgos.

Cinco dos seis maiores campos de musgos localizam-se na costa voltada para o estreito de Drake. Isso é atribuído ao vento predominante Noroeste e Oeste que nos meses de verão traz umidade do mar para essa área da península Fildes. As praias voltadas para o estreito de Drake são também aquelas que apresentam maior presença de animais, produtores de matéria orgânica que promove transferência de nutrientes para essas áreas, contribuindo para crescimento da vegetação.

Mesmo com o resfriamento observado para algumas áreas da Península Antártica no período 1999 - 2014, a ilha Rei George apresenta aumento de temperatura do ar, e com isso espera-se crescimento da vegetação da península Fildes nos próximos anos, com contínua retração da geleira Collins e aumento da disponibilidade de água líquida no sistema.

\section{Referências}

Amesbury, M. J., Roland, T. P., Royles, J., Hodgson, D. A., Convey, P., Griffiths, H. \& Charman, D. J. (2017). Widespread biological response to rapid warming on the Antarctic Peninsula. Current Biology, 27(11), 1.616-1.622. https://doi. org/10.1016/j.cub.2017.04.034

Andrade, A. M., Michel, R. F. M., Bremer, U. F., Schaefer, C. E. R. G. \& Simões, J. C. (2018). Relationship between solar radiation and surface distribution of vegetation in Fildes Peninsula and Ardley Island, Maritime Antarctica. International Journal of Remote Sensing, 39(8), 2.238-2.254. https://doi.org/10 $.1080 / 01431161.2017 .1420937$

Andrade, A. M., Michel, R. F. M., Fonseca, E. L., Bremer, U. F., Schaefer, C. E. R. G. \& Simões, J. C. 
(2015). Influência da radiação solar na distribuição superficial da vegetação na Península Fildes, Antártica Marítima. Anais XVII Simpósio Brasileiro de Sensoriamento Remoto - SBSR, João Pessoa-PB, Brasil. Recuperado de https://www.researchgate.net/ publication/281443559_Influencia_da_radiacao_solar_na_distribuicao_superficial_da_vegetacao_na_ Peninsula_Fildes_Antartica_Maritima

Billings W.D. \& Morris R. J. (1951). Reflection of visible and infrared radiation from leaves of different ecological groups. American Journal of Botany, 38(5), 327-331. https://doi.org/10.2307/2437820

Bubier, J. L., Rock, B. N., \& Crill, P. M. (1997). Spectral reflectance measurements of boreal wetland and forest mosses. Journal of Geophysical Research: Atmospheres, 102(D24), 29.483-29.494. https://doi. org/10.1029/97jd02316

Braun, M.H., Betsch, T., \& Seehaus, T. (2016). King George Island TanDEM-XDEM, link to GeoTIFF. Institut für Geographie, Friedrich-Alexander-Universität, Erlangen-Nürnberg, PANGAEA. https://doi. org/10.1594/PANGAEA.863567

Braun, M. \& Gossmann, H. (2002). Glacial changes in the area of Admiralty Bay and Potter Cove, King George Island, Antarctica. En L. Beyer \& M. Bölter (Eds.), Geoecology of Antarctic Ice-Free Coastal Landscapes (pp. 75-89). Berlin-Heidelberg: SpringerVerlag.

Bremer, U. F. (1998). Morfologia e bacias de drenagem na cobertura de gelo da ilha Rei George, Antártica. (Disertación de Maestría, Universidade Federal do Rio Grande do Sul, Porto Alegre, Brasil). Recuperada de http://hdl.handle.net/10183/1395

Bremer, U. F. (2008). Soils and geomorphology of the Eastern Warszawa Peninsula, King George Island, maritime Antarctica. (Tesis de Doctorado, Universidade Federal de Viçosa, Viçosa, Brasil). Recuperada de http://hdl.handle.net/10183/30989

Casanovas, P., Black, M., Fretwell, P., \& Convey, P. (2015). Mapping lichen distribution on the Antarctic Peninsula using remote sensing, lichen spectra and photographic documentation by citizen scientists. $P o-$ lar Research, 34, 1-9. https://doi.org/10.3402/polar. v34.25633
Chilar, J. (2000). Land cover mapping of large areas from satellites: Status and research priorities. International Journal of Remote Sensing, 21(6-7), 1.0931.114. https://doi.org/10.1080/014311600210092

Convey, P. \& Smith, R.I.L. (2006). Responses of terrestrial Antarctic ecosystems to climate change. Plant Ecology, 182(1-2),1-10. https://doi.org/10.1007/ s11258-005-9022-2

Convey, P. (2003). Maritime Antarctic climate change: signals from terrestrial biology. En E. Domack, A. Levente, A. Burnet, R. Bindschadler, P. Convey y M. Kirby (Eds.), Antarctic Peninsula Climate Variability. Historical and Paleoenvironmental Perspectives (pp. 145-158). https://doi.org/10.1029/AR079p0145

Cook, A.J., Fox, A.J., Vaughan, D.G., \& Ferrigno, J.G. (2005) Retreating glacier fronts on the Antarctic Peninsula over the past half-century. Science, 308(5721), 541-544. https://doi.org/10.1126/science.1104235

Cook, A.J., Holland, P.R., Meredith, M.P., Murray, T., Luckman, A. \& Vaughan, D.G. (2016). Ocean forcing of glacier retreat in the western Antarctic Peninsula. Science, 353(6296), 283-286. https://doi.org/10.1126/ science.aae 0017

Fretwell, P.T., Convey, P., Fleming, A.H., Peat, H.J., \& Hughes, K.A. (2011). Detecting and mapping vegetation distribution on the Antarctic Peninsula from remote sensing data. Polar Biology, 34(2), 273-281. https://doi.org/10.1007/s00300-010-0880-2

Gerighausen, U., Bräutigam, K., Mustafa, O. \& Peter H.U. (2003). Expansion of vascular plants on an Antarctic island; a consequence of climate change? Antarctic biology in a global context; proceedings of the VIIIth SCAR international biology symposium, Netherlands, 8, 79-83. https://doi.org/10.13140/ RG.2.1.4727.8487

Green, A.T.G., Sancho, L.G., Pintado, A. \& Schroeter, B. (2011). Functional and spatial pressures on terrestrial vegetation in Antarctica forced by global warming. Polar Biology, 34, 1643-1656. https://doi. org/10.1007/s00300-011-1058-2

Haselwimmer, C., \& Fretwell, P. (2009). Field reflectance spectroscopy of sparse vegetation cover on the Antarctic Peninsula. First Workshop on Hyperspectral Image and Signal Processing: Evolution in Re- 
mote Sensing, Piscataway, NJ. Recuperado de https:// doi.org/10.1109/WHISPERS.2009.5289099

Hochschild, V. \& Staeblein, G. (1998). Geomorpholgische Kartierung der Potter Halbinsel (King George Island) mitoptischen Fernerkundungsdaten und ERS1-SAR-Daten. En Goßmann H (Ed.), Patagonien und Antarktis - Geofernerkundungmit ERS-1-Radarbildern (pp. 101-117). Gotha: Perthes.

Jawak, S.D., Udhayaraj, A.D. \& Alvarinho, J. L. (2016). Geospatial mapping of vegetation in the Antarctic environment using very high-resolution WorldView-2 imagery. Proc. SPIE 9877, Land Surface and Cryosphere Remote Sensing III, 98772N. https://doi. org/10.1117/12.2222751

Johansen, B. \& Karlsen, S.R. (2005). Monitoring vegetation changes on Finnmarksvidda, Northern Norway, using Landsat MSS and Landsat TM/ETM+ satellite images. Phytocoenologia, 35(4), 969-984. https://doi.org/10.1127/0340-269X/2005/0035-0969

John, B. S. (1972). Evidence from the South Shetland Islands towards a glacial history of West Antarctica. Institute of British Geographers, Special Publication, $4,75-89$.

Kejna, M., Araźny, A., \& Sobota, I. (2013). Climatic change on King George Island in the years 19482011. Polish Polar Research, 34(2), 213-235. https://doi.org/10.2478/popore-2013-0004

Knipling, E.B. (1970). Physical and physiological basis for the reflectance of visible and near-infrared radiation from vegetation. Remote Sensing of Environment, 1(3), 155-159. https://doi.org/10.1016/ S0034-4257(70)80021-9

López-Martínez, J., Serrano, E., Schmid, T., Mink, S., \& Linés, C. (2012). Periglacial processes and landforms in the South Shetland Islands (northern Antarctic Peninsula region). Geomorphology, 155-156, 62-79. https://doi.org/10.1016/j.geomorph.2011.12.018

Lucieer, A., Robinson, S., Turner, D., Harwin, S., \& Kelcey, J. (2012).Using a micro-uav for ultra-high resolution multi-sensor observations of antarctic moss beds. ISPRS - International Archives of the Photogrammetry, Remote Sensing and Spatial In- formation Sciences, 39-B1, 429-433. http://dx.doi. org/10.5194/isprsarchives-XXXIX-B1-429-2012

Mäusbacher, R. (1991) Die Jungkvartare Relief - und Klimageschichte im Bereich der Fildeshalbinsel, Süd-Shetland-Inseln. Antarktis: Heidelberger.

Michel, R.F.M., Schaefer, C.E.G.R., López-Martínez, J., Simas, F.N.B., Haus, N.W., Serrano, E. \& Bockheim, J.G. (2014) Soils and landforms from Fildes Peninsula and Ardley Island, Maritime Antarctica. Geomorphology, 225, 76-86. https://doi.org/10.1016/j. geomorph.2014.03.041

Mulvaney, R., Abram, N.J., Hindmarsh, R.C.A., Arrowsmith, C., Fleet, L., Triest, J., ... Foord, S. (2012). Recent Antarctic Peninsula warming relative to Holocene climate and ice-shelf history. Nature, 489(7414), 141-144. https://doi.org/10.1038/nature11391

Olech, M. A. \& Massalski, A. (2001). Plant colonization and community development on the Sphinx Glacier forefield. Folia Facultatis Scientiarium Naturalium Universitatis Masarykianae Brunensis, Geographia, 25, 111-119.

Osmanoğlu, B., Braun, M., Hock, R., \& Navarro, F.J. (2013). Surface velocity and ice discharge of the ice cap on King George Island, Antarctica. Annals of Glaciology, 54(63), 111-119. https://doi. org/10.3189/2013AoG63A517

Park, B-K., Chang, S-K., Yoon, H. I., \& Chung, H. (1998). Recent retreat of ice cliffs, King George Island, South Shetland Islands, Antarctic Peninsula. Annals of Glaciology, 27, 633-635. https://doi.org/10.3189/1998AoG27-1-633-635

Petsch, C. (2018). Evolução hidro geomorfológica da zona proglacial da Geleira Collins, Ilha Rei George, Antártica (Tesis de Doctorado, Universidade Federal do Rio Grande do Sul, Porto Alegre, Brasil). Recuperado de https://lume.ufrgs.br/handle/10183/178701

Peter H. U., Bußer, C., Mustafa, O., \& Pfeiffer, S. (2007). Risk assessment for the Fildes Peninsula and Ardley Island and the development of management plans for designation as Antarctic Specially Protected or Managed Areas. Germany: Federal Environment Agency. 
Poelking, E.L., Andrade, A.M., Schaefer, C.E.G.R., Simas, F.N.B., \& Fernandes Filho, E.I. 2011). Vegetation Map of Potter Peninsula, Maritime Antarctica. Trabajo presentado en XV Simpósio Brasileiro de Sensoriamento Remoto, Curitiba, PR. Recuperado de https://www.researchgate.net/publication/267802816_Vegetation_Map_of_Potter_Peninsula_Maritime_Antarctica

Pritchard, H.D. \& Vaugan, D.G. (2007). Widespread acceleration of tidewater glaciers on the Antarctic Peninsula. Journal of Geophysical Research, 112(F3), 1-10. https://doi.org/10.1029/2006JF000597

Pudelko, R., Angiel, P.J., Potocki, M., Jedrejek, A., \& Kozak, M. (2018). Fluctuation of glacial retreat rates in the eastern part of Warszawa icefield, King George Island, Antarctica, 1979-2018. Remote Sensing, 10(6), 892. https://doi.org/10.3390/ rs 10060892

Putzke, J., Athanásio, C.G., de Albuquerque, M.G., Victoria, F.C., \& Pereira, A.B (2015). Comparative study of moss diversity in South Shetland Islands and in the Antarctic Peninsula. Revista Chilena de Historia Natural, 88(6), 1-6. https://doi. org/10.1186/s40693-014-0033-z

Rakusa-Suszczewski, S. (2003). Functioning of the geoecosystem for the west side of Admiralty Bay (King George Island, Antarctica): Outline of research at Arctowski Station. Ocean and Polar Research, 25(4), 653-662. https://doi.org/10.4217/OPR.2003.25.4.653

Rosa, K.K., Petsch, C., Campana, A.S., Simões, C.L., Gonçalves, M.A., \& Viel, J.A. (2015). Evolução de ambientes proglaciais em resposta às mudanças climáticas. Anais do XI Simpósio Nacional de Geomorfologia. Maringá, Brasil. Recuperado de http://www. sinageo.org.br/2016/trabalhos/4/4-252-45.html

Rosa, K., Vieira, R., Ferrando, F., \& Simões, J. (2009). Feições sedimentológicas e geomorfológicas do ambiente de deglaciação das geleiras Wanda e Ecology, ilha Rei George, Antártica. Pesquisas em Geociências, 36(3), 315-326.

Ruiz-Fernández, J., Oliva, B., \& García-Hernández, C. (2017). Topographic and geomorphologic controls on the distribution of vegetation formations in Elephant
Point (Livingston Island, Maritime Antarctica). Science of The Total Environment, 587-588, 340-349. https://doi.org/10.1016/j.scitotenv.2017.02.158

Rückamp, M., Braun, M., Suckro, S., \& Blindow, N. (2011). Observed glacial changes on the King George Island ice cap, Antartica, in the last decade. Global and Planetary Change, 79(1-2), 99-109. https://doi. org/10.1016/j.gloplacha.2011.06.009

Schaefer, C.E.G.R., Simas, F.B.N, Albuquerque-Filho, M.R., Michel, R.F.M, Viana, J. \&Tatur, H.M. (2004). Fosfatização: processo de formação de solos na Baía do Almirantado e implicações ambientais. En C.E. G.R. Schaefer, R. Francelino, F.N.B. Simas, R. Albuquerque Filho (Eds.). Ecossistemas costeiros e monitoramento ambiental da Antártica Marítima: Baia do Almirantado, Ilha Rei George (pp. 47-59). Viçosa: NEPUT-Departamento de Solos.

Serrano, E. (2003). Paisaje natural y pisos geoecológicos en las áreas libres de hielo de la Antártida marítima (Islas Shetland del Sur). Boletín de la Asociación de Geógrafos Españoles, 35, 5-32.

Simas, F.N.B., Schaefer, C.E.G.R., Filho, M.R.A., Francelino, M.R., Filho, E.I.F., \& da Costa, L.M. (2008). Genesis, properties and classification of Cryosols from Admiralty Bay, maritime Antarctica. Geoderma, 144, (1-2), 116-122. https://doi.org/10.1016/j. geoderma.2007.10.019

Simões, J.C., Bremer, U.F., Aquino, F.E., \& Ferron, F.A. (1999). Morphology and variations of glacial drainage basins in King George Island ice field, Antarctica. Annals of Glaciology, 29, 220-224. https://doi. org/10.3189/172756499781821085

Simões, C.L., da Rosa, K.K., Czapela, F.F., Vieira, R. \& Simões, J.C. (2015). Collins Glacier retreat process and regional climatic variations, King George Island, Antarctica. Geographical Review, 105(4), 462-471. https://doi.org/10.1111/j.1931-0846.2015.12091.x

Singh, J., Singh, R.P., \& Khare, R. (2018). Influence of climate change on Antarctic flora. Polar Science, 18 94-101. https://doi.org/10.1016/j.polar.2018.05.006

Smith, R. I. L. (2001). Plant colonisation response to climate change in the Antarctic. Geographia, 25, 19-33. 
Steig, E. J., Schneider, D. P., Rutherford, S. D., Mann, M.E., Comiso, J.C., \& Shindell, D.T. (2009). Warming of the Antarctic ice-sheet surface since the 1957 International Geophysical Year. Nature, 457(7228), 459-462. https://doi.org/10.1038/nature07669

Sotille, M. E., Bremer, U. F., \& Simões, J. C. (2017). Uso de NDVI de imágenes de vehículo aéreo no tripulado (VANT) para mapeo de vegetación en Bahía Esperanza, P. Antártica. Trabajo presentado en el IX Congreso Latinoamericano de Ciencia Antártica, Punta Arenas.

Turner, J., Colwell, S.R., \& Harangozo, S. A. (1997). Variability of precipitation over the coastal western Antarctic Peninsula from synoptic observations. Journal of Geophysical Research, 102(D12), 13.99914.007. https://doi.org/10.1029/96JD03359

Turner, J., Lu, H., White, I., King, J.C., Phillips, T., Hosking, J.S., ... Deb, P. (2016). Absence of 21st century warming on Antarctic Peninsula consistent with natural variability. Nature, 535(7612), 411-415. https://doi.org/10.1038/nature18645

Vera, M. L., Fernández-Teruel, T., \& Quesada, A. (2013). Distribution and reproductive capacity of Deschampsia antarctica and Colobanthus quitensis on Byers Peninsula, Livingston Island, South Shetland Islands, Antarctica. Antarctic Science, 25 (2), 292302. https://doi.org/10.1017/S0954102012000995

Victoria, F. de C., de Albuquerque, M.P., Pereira, A.B., Simas, F.N.B., Spielmann, A.A., \& Schaefer, C.E. G.R. (2013). Characterization and mapping of plant communities at Hennequin Point, King George Island, Antarctica. Polar Research, 32, 19.261. https:// doi.org/10.3402/polar.v32i0.19261

\section{Agradecimentos}

Esta investigação foi financiada pelo Conselho Nacional de Desenvolvimento Científico e Tecnológico (CNPq) e a Fundação de Amparo à Pesquisa do Estado do Rio Grande do Sul (FAPERGS) por um auxílio ao Instituto Nacional da Ciência e Tecnologia da Criosfera (INCT da Criosfera). Carina Petsch agradece a Coordenação de Aperfeiçoamento de Pessoal de Nível Superior (CAPES) por uma bolsa de estudo. Os dados de campo são provenientes do projeto: "Estudos da variabilidade climática e ambiental nas Ilhas Shetland do Sul e Península Antártica por meio de registros sedimentares terrestres e marinhos - Chamada No 64/2013 - MCTI/CNPq/FNDCT-Ação Transversal - Programa Antártico Brasileiro - PROANTAR" sob coordenação de Rosemary Vieira. 